\title{
Strong Convergence Theorems for a Common Fixed Point of a Finite Family of Bregman Weak Relativity Nonexpansive Mappings in Reflexive Banach Spaces
}

\author{
Habtu Zegeye ${ }^{1}$ and Naseer Shahzad ${ }^{2}$ \\ ${ }^{1}$ Department of Mathematics, University of Botswana, Private Bag 00704, Gaborone, Botswana \\ ${ }^{2}$ Department of Mathematics, King Abdulaziz University, P.O. Box 80203, Jeddah 21589, Saudi Arabia \\ Correspondence should be addressed to Naseer Shahzad; nshahzad@kau.edu.sa
}

Received 28 August 2013; Accepted 22 January 2014; Published 13 March 2014

Academic Editors: L. B. Ćirić, B. Lamichhane, T. Li, A. Moudafi, and C. Zhai

Copyright ( $\odot 2014$ H. Zegeye and N. Shahzad. This is an open access article distributed under the Creative Commons Attribution License, which permits unrestricted use, distribution, and reproduction in any medium, provided the original work is properly cited.

We introduce an iterative process for finding an element of a common fixed point of a finite family of Bregman weak relatively nonexpansive mappings. Our theorems improve and unify most of the results that have been proved for this important class of nonlinear operators.

\section{Introduction}

Throughout this paper, $f: E \rightarrow(-\infty,+\infty]$ is a proper, lower semicontinuous, and convex function, where $E$ is a real reflexive Banach space with $E^{*}$ as its dual. Denote the domain of $f$ by $\operatorname{dom} f$; that is, $\operatorname{dom} f=\{x \in E: f(x)<\infty\}$. Let $f$ : $E \rightarrow(-\infty,+\infty]$ be a Gâteaux differentiable (see Section 2) function. The function $D_{f}: \operatorname{dom} f \times \operatorname{int}(\operatorname{dom} f) \rightarrow[0,+\infty)$ defined by

$$
D_{f}(x, y):=f(x)-f(y)-\langle\nabla f(y), x-y\rangle,
$$

where $\nabla f$ is the gradient of $f$, is called the Bregman distance with respect to $f$ [1]. The following property of Bregman distance function is known:

$$
\begin{array}{r}
D_{f}(x, z)-D_{f}(x, y)-D_{f}(y, z) \\
=\langle\nabla f(y)-\nabla f(z), x-y\rangle,
\end{array}
$$

for $x \in \operatorname{dom} f$ and $y, z \in \operatorname{int}(\operatorname{dom} f)(\operatorname{see}[1,2])$.

A Bregman projection with respect to $f$ [1] of $x \in$ $\operatorname{int}(\operatorname{dom} f)$ onto the nonempty closed and convex set $C \subset$ $\operatorname{dom} f$ is the unique vector $P_{C}^{f}(x) \in C$ satisfying

$$
D_{f}\left(P_{C}^{f}(x), x\right)=\inf \left\{D_{f}(y, x): y \in C\right\} .
$$

Remark 1. If $E$ is a smooth Banach space, setting $f(x)=\|x\|^{2}$, for all $x \in E$ we have $\nabla f(x)=2 J x$, for all $x \in E$, where $J$ is the normalized duality mapping from $E$ onto $E^{*}$, and hence we have the following.

(i) $D_{f}(x, y)$ reduces to $D_{f}(x, y)=\|x\|^{2}-2\langle x, J y\rangle+$ $\|y\|^{2}=\phi(x, y)$, for all $x, y \in E$, which is the Lyapunov function introduced by Alber [3]. If $E=$ $H$, a Hilbert space, $J$ is identity mapping and hence $D_{f}(x, y)$ becomes $D_{f}(x, y)=\|x-y\|^{2}$, for $x, y \in H$.

(ii) The Bregman projection $P_{C}^{f}(x)$ reduces to the generalized projection $\Pi_{C}(x)$ (see, e.g., [3]) which is defined by

$$
\phi\left(\Pi_{C}(x), x\right)=\min _{y \in C} \phi(y, x) .
$$

Let $C$ be a nonempty and convex subset of $\operatorname{int}(\operatorname{dom} f)$ and let $T: C \rightarrow \operatorname{int}(\operatorname{dom} f)$ be a mapping. A mapping $T: C \rightarrow$ $C$ is said to be nonexpansive if $\|T x-T y\| \leq\|x-y\|$, for all $x, y \in$ $C$. $T$ is said to be quasi-nonexpansive if $F(T) \neq \emptyset$ and $\| T x-$ $p\|\leq\| x-p \|$, for all $x \in C$ and $p \in F(T)$, where $F(T)$ stands for the fixed point set of $T$; that is, $F(T)=\{x \in C: T x=x\}$. A point $p \in C$ is called an asymptotic fixed point of $T$ (see [4]) 
if $C$ contains a sequence $\left\{x_{n}\right\}$ which converges weakly to $p$ such that $\lim _{n \rightarrow \infty}\left\|x_{n}-T x_{n}\right\|=0$. We denote by $\widehat{F}(T)$ the set of asymptotic fixed points of $T$. A point $p \in C$ is called a strong asymptotic fixed point of $T$ if there exists a sequence $\left\{x_{n}\right\}$ in $C$ which converges strongly to $p$ and $\lim _{n \rightarrow \infty}\left\|x_{n}-T x_{n}\right\|=0$. We denote the set of all strong asymptotic fixed points of $T$ by $\widetilde{F}(T)$.

A mapping $T: C \rightarrow \operatorname{int}(\operatorname{dom} f)$ with $F(T) \neq \emptyset$ is called

(i) Bregman quasi-nonexpansive [5] if

$$
D_{f}(p, T x) \leq D_{f}(p, x), \quad \forall x \in C, p \in F(T) ;
$$

(ii) Bregman relatively nonexpansive [5] if

$$
\begin{gathered}
D_{f}(p, T x) \leq D_{f}(p, x), \quad \forall x \in C, \\
p \in F(T), \quad \widehat{F}(T)=F(T)
\end{gathered}
$$

(iii) Bregman firmly nonexpansive [6] if, for all $x, y \in C$,

$$
\begin{aligned}
& \langle\nabla f(T x)-\nabla f(T y), T x-T y\rangle \\
& \quad \leq\langle\nabla f(x)-\nabla f(y), T x-T y\rangle ;
\end{aligned}
$$

or, equivalently,

$$
\begin{aligned}
& D_{f}(T x, T y)+D_{f}(T y, T x)+D_{f}(T x, x)+D_{f}(T y, y) \\
& \leq D_{f}(T x, y)+D_{f}(T y, x) .
\end{aligned}
$$

(iv) Bregman weak relatively nonexpansive if

$$
\begin{gathered}
D_{f}(p, T x) \leq D_{f}(p, x), \quad \forall x \in C, \\
p \in F(T), \quad \widetilde{F}(T)=F(T) .
\end{gathered}
$$

Remark 2. We observe from the above definitions that every relatively nonexpansive mapping $T$ is Bregman relatively nonexpansive mapping with respect to $f(x)=\|x\|^{2}$, for all $x \in E$, where $T$ is called relatively nonexpansive mapping if the following conditions are satisfied:

$$
\begin{aligned}
& \phi(p, T x) \leq \phi(p, x), \quad \forall x \in C, \\
& p \in F(T), \quad \widehat{F}(T)=F(T) \neq \emptyset .
\end{aligned}
$$

If $E=H$, a real Hilbert space, then relatively nonexpansive mappings are demiclosed quasi-nonexpansive mappings which include the class of nonexpansive mappings with fixed point nonempty.

Remark 3. It is shown in [6] that if $T$ is Bregman firmly nonexpansive then $\widehat{F}(T)=\widetilde{F}(T)=F(T)$ and hence it is Bregman relatively nonexpansive provided that the Legendre function $f$ is uniformly Fréchet differentiable and bounded on bounded sets of $E$.

Remark 4. We observe from the above facts that the class of Bregman weak relatively nonexpansive mappings includes the class of Bregman relatively nonexpansive mappings and hence the class of Bregman firmly nonexpansive mappings. In addition, we also have that every continuous Bregman quasinonexpansive is Bregman weak relatively nonexpansive mapping.

The following example by Chen et al. [7] shows that the inclusion is proper.

Example 5. Let $E=l^{2}, f(x)=(1 / 2)\|x\|^{2}$, for all $x \in E$. Let $\left\{x_{n}\right\} \subset E$ be defined by $x_{0}=(1,0,0, \ldots), x_{1}=(1,1,0,0, \ldots)$, $x_{2}=(1,0,1, \ldots) \ldots, x_{n}=\left(\xi_{n, 1}, \xi_{n, 2}, \ldots, \xi_{n, k}, \ldots\right), \ldots$, where

$$
\xi_{n, k}:= \begin{cases}1, & \text { if } k=1, n+1 \\ 0, & \text { otherwise } \forall n \geq 1\end{cases}
$$

Let $T: E \rightarrow E$ be defined by

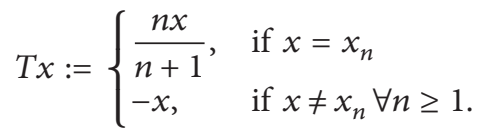

Then, it is shown in [7] that $T$ is Bregman weak relatively nonexpansive but not Bregman relatively nonexpansive.

Construction of fixed points of nonexpansive mappings and relatively nonexpansive mappings and their generalizations is an important subject in nonlinear operator theory and its applications, in particular, in image recovery and signal processing (see, e.g., [8-14] and the references therein). Mann [15] and Ishikawa [16] iteration process for approximating fixed point iteration process for nonexpansive mappings and relatively nonexpansive mappings in Hilbert spaces and Banach spaces have been studied extensively by many authors to solve nonlinear operator equations as well as variational inequalities (see, e.g., [15-21]). However, both iteration processes have only weak convergence even in Hilbert spaces (see, e.g., $[15,16])$. Some attempts to modify the Mann iteration method so that strong convergence is guaranteed have been made.

In 2003, Nakajo and Takahashi [22] proposed the following modification of the Mann iteration method for a nonexpansive mapping $T: C \rightarrow C$ in a Hilbert space $H$ :

$$
\begin{gathered}
x_{0} \in C, \text { chosen arbitrarily, } \\
y_{n}=\alpha_{n} x_{n}+\left(1-\alpha_{n}\right) T x_{n}, \\
C_{n}=\left\{z \in C:\left\|z-y_{n}\right\| \leq\left\|z-x_{n}\right\|\right\}, \\
Q_{n}:=\left\{z \in C:\left\langle x_{n}-z, x_{n}-x_{0}\right\rangle \leq 0\right\}, \\
x_{n+1}=P_{C_{n} \cap Q_{n}}^{f}\left(x_{0}\right), \quad \forall n \geq 0,
\end{gathered}
$$

where $\left\{\alpha_{n}\right\} \subset[0,1]$ and $P_{C}$ denotes the metric projection from $H$ onto a closed convex subset $C$ of $H$. They proved that the sequence $\left\{x_{n}\right\}$ defined by (13) converges strongly to a fixed point of $T$ under some suitable conditions.

In 2005, Matsushita and Takahashi [23] introduced the following modification of the Mann iteration method for a 
relatively nonexpansive mapping $T: C \rightarrow C$ in a Banach space $E$ as follows:

$$
\begin{gathered}
x_{0} \in C, \text { chosen arbitrarily, } \\
y_{n}=J^{-1}\left(\alpha_{n} J x_{n}+\left(1-\alpha_{n}\right) J T x_{n}\right), \\
C_{n}=\left\{z \in C: \phi\left(z, y_{n}\right) \leq \phi\left(z, x_{n}\right)\right\}, \\
Q_{n}:=\left\{z \in C:\left\langle x_{n}-z, J x_{n}-J x_{0}\right\rangle \leq 0\right\}, \\
x_{n+1}=\prod_{C_{n} \cap Q_{n}}^{f}\left(x_{0}\right), \quad \forall n \geq 0,
\end{gathered}
$$

where $\left\{\alpha_{n}\right\} \subset[0,1]$ and $\phi(y, x)$ is as shown in Remark 1 . They showed that $\left\{x_{n}\right\}$ generated by (14) converges strongly to a fixed point of $T$ under some suitable assumptions.

In [24], Reich and Sabach proposed an algorithm for finding a common fixed point of finitely many Bregman firmly nonexpansive mappings $T_{i}: C \rightarrow C(i=1,2, \ldots, N)$ satisfying $\bigcap_{i=1}^{N} F\left(T_{i}\right) \neq \emptyset$ in a reflexive Banach space $E$ as follows:

$$
\begin{gathered}
x_{0} \in E, \text { chosen arbitrarily, } \\
Q_{0}^{i}=E, \\
y_{n}^{i}=T_{i}\left(x_{n}+e_{n}^{i}\right), \\
Q_{n+1}^{i}=\left\{z \in Q_{n}^{i}:\left\langle\nabla f\left(x_{n}+e_{n}^{i}\right)-\nabla f\left(y_{n}^{i}\right), z-y_{n}^{i}\right\rangle \leq 0\right\}, \\
Q_{n}=\bigcap_{i=1}^{N} Q_{n}^{i}, \\
x_{n+1}=P_{Q_{n+1}}^{f}\left(x_{0}\right), \quad \forall n \geq 0 .
\end{gathered}
$$

They proved that, under suitable conditions, the sequence $\left\{x_{n}\right\}$ generated by (15) converges strongly to $\bigcap_{i=1}^{N} F\left(T_{i}\right)$ and applied it to the solution of convex feasibility and equilibrium problems. You may also see $[5,25]$.

Inspired and motivated by the above works, Chen et al. [7] proposed an algorithm for finding a fixed point of Bregman weak relatively nonexpansive mapping $T_{i}: C \rightarrow C(i=$ $1,2, \ldots, N)$ satisfying $\mathscr{F}:=\bigcap_{i=1}^{N} F\left(T_{i}\right) \neq \emptyset$ in a reflexive Banach space $E$ as follows:

$$
\begin{gathered}
x_{0} \in C, \quad Q_{0}=C, \text { chosen arbitrarily, } \\
z_{n}=\nabla f^{*}\left(\beta_{n} \nabla f\left(T\left(x_{n}+e_{n}\right)\right)+\left(1-\beta_{n}\right) \nabla f\left(x_{n}+e_{n}\right)\right) ; \\
y_{n}=\nabla f^{*}\left(\alpha_{n} \nabla f\left(T\left(x_{n}+e_{n}\right)\right)+\left(1-\alpha_{n}\right) \nabla f\left(z_{n}\right)\right) ; \\
C_{n}=\left\{z \in C_{n-1} \cap Q_{n-1}: D_{f}\left(z, y_{n}\right) \leq D_{f}\left(z, x_{n}+e_{n}\right)\right\}, \\
C_{0}=\left\{z \in C: D_{f}\left(z, y_{0}\right) \leq D_{f}\left(z, x_{0}\right)\right\}, \\
\left.Q_{n} \quad \forall z \in C_{n-1} \cap Q_{n-1}:\left\langle\nabla f\left(x_{0}\right)-\nabla f\left(x_{n}\right), z-x_{n}\right\rangle \leq 0\right\}, \\
x_{n+1}=P_{C_{n} \cap Q_{n}}^{f}\left(x_{0}\right), \quad \forall n \geq 0,
\end{gathered}
$$

where $\left\{\alpha_{n}\right\},\left\{\beta_{n}\right\} \subset[0,1]$ satisfy certain conditions and $\left\{e_{n}\right\}$ is an error sequence in $E$ with $e_{n} \rightarrow 0$ as $n \rightarrow \infty$. They proved that, under suitable conditions, the sequence $\left\{x_{n}\right\}$ generated by (16) converges strongly to $p=P_{F(T)}^{f}\left(x_{0}\right)$, where $P_{F(T)}^{f}\left(x_{0}\right)$ is the Bregman projection of $C$ onto $F(T)$.

Moreover, in [26], Naraghirad and Yao introduced a hybrid iteration algorithm for finding a common fixed point of infinite family of Bregman weak relatively nonexpansive mapping $T_{i}: C \rightarrow C(i=1,2, \ldots)$ provided that $\mathscr{F}:=\bigcap_{i=1}^{\infty} F\left(T_{i}\right) \neq \emptyset$, in a reflexive Banach space $E$. They proved that, under suitable conditions, their hybrid iteration algorithm converges strongly to $\mathscr{F}$.

Remark 6. It is worth mentioning that all the iteration algorithms introduced and used above seem cumbersome and complicated in the sense that at each stage of the iteration computations of the set(s) $C_{n}$ and/or $Q_{n}$ are required and the next iterate is taken as the Bergman projection of $x_{0}$ on the intersection of $C_{n}$ and/or $Q_{n}$. This seems difficult to do in applications.

It is our purpose in this paper to introduce an iterative algorithm for finding a common fixed point of a finite family of Bregman weak relatively nonexpansive mappings in reflexive Banach spaces. We prove strong convergence theorem for the sequence produced by the method. Our scheme does not require computations of the set $C_{n}$ or $Q_{n}$ at each stage of iterates. We prove strong convergence theorems for the sequences produced by the method. Our results improve and generalize many known results in the current literature (see, e.g., $[7,23,24]$ ).

\section{Preliminaries}

Let $f: E \rightarrow(-\infty,+\infty]$ be a function. For any $x \in \operatorname{Dom}(f)$ and $y \in E$, the right-hand derivative of $f$ at $x$ in the direction of $y$ is defined by $f^{\circ}(x, z)=\lim _{t \rightarrow 0^{+}}(f(x+t y)-f(x)) / t$. The function $f$ is said to be Gâteaux differentiable at $x$ if $\lim _{t \rightarrow 0^{+}}(f(x+t y)-f(x)) / t$ exists for any $y$. In this case, $f^{\circ}(x, y)$ coincides with $\nabla f(x)$, the value of the gradient $\nabla f$ of $f$ at $x$. The function $f$ is said to be Gâteaux differentiable if it is Gâteaux differentiable for any $x \in \operatorname{int}(\operatorname{dom} f)$. The function $f$ is said to be Fréchet differentiable at $x$ if this limit is attained uniformly in $\|y\|=1$ and $f$ is said to be uniformly Fréchet differentiable on a subset $C$ of $E$ if the limit is attained uniformly for $x \in C$ and $\|y\|=1$.

Let $x \in \operatorname{int}(\operatorname{dom} f)$. The subdifferential of $f$ at $x \in E$ is the convex set defined by

$$
\partial f(x)=\left\{x^{*} \in E^{*}: f(x)+\left\langle x^{*}, y-x\right\rangle \leq f(y), \forall y \in E\right\} .
$$

The Fenchel conjugate of $f$ is the function $f^{*}: E^{*} \rightarrow$ $(-\infty,+\infty]$ defined by $f^{*}\left(x^{*}\right)=\sup \left\{\left\langle x^{*}, x\right\rangle-f(x): x \in E\right\}$.

The function $f$ is said to be essentially smooth if $\partial f$ is both locally bounded and single-valued on its domain. It is called essentially strictly convex, if $(\partial f)^{-1}$ is locally bounded on its domain and $f$ is strictly convex on every convex subset of $\operatorname{dom} \partial f$. $f$ is said to be a Legendre, if it is both 
essentially smooth and essentially strictly convex. When the subdifferential of $f$ is single-valued, it coincides with the gradient $\partial f=\nabla f$ (see [27]).

For a Legendre function $f$ the following properties are known.

(i) $f$ is essentially smooth if and only if $f^{*}$ is essentially strictly convex (see [28, Theorem 5.4]).

(ii) One has $(\partial f)^{-1}=\partial f^{*}$ (see [29]).

(iii) $f$ is Legendre if and only if $f^{*}$ is Legendre (see [28, Corollary 5.5]).

(iv) If $f$ is Legendre, then $\nabla f$ is a bijection satisfying $\nabla f=\left(\nabla f^{*}\right)^{-1}, \operatorname{ran} \nabla f=\operatorname{dom} \nabla f^{*}=\operatorname{int} \operatorname{dom} f^{*}$, and $\operatorname{ran} \nabla f^{*}=\operatorname{dom} \nabla f=\operatorname{int} \operatorname{dom} f$ (see [28, Theorem 5.10]).

When $E$ is a smooth and strictly convex Banach space, one important and interesting example of Legendre function is $f(x):=(1 / p)\|x\|^{p}(1<p<\infty)$. In this case the gradient $\nabla f$ of $f$ coincides with the generalized duality mapping of $E$; that is, $\nabla f=J_{p}(1<p<\infty)$. In particular, $\nabla f=I$, the identity mapping in Hilbert spaces.

A function $f$ on $E$ is coercive [30] if the sublevel set of $f$ is bounded; equivalently, $\lim _{\|x\| \rightarrow \infty} f(x)=\infty$. A function $f$ on $E$ is said to be strongly coercive [31] if $\lim _{\|x\| \rightarrow \infty} f(x) /\|x\|=$ $\infty$.

Let $B_{r}:=\{z \in E:\|z\| \leq r\}$, for all $r>0$, and $S_{E}=\{x \in E$ : $\|x\|=1\}$. Then, a function $f: E \rightarrow \mathbb{R}$ is said to be uniformly convex on bounded subsets of $E$ ([31, pp. 203]) if $\rho_{r}(t)>0$, for all $r, t>0$, where $\rho_{r}:[0, \infty) \rightarrow[0, \infty]$ is defined by

$$
\begin{aligned}
\rho_{r}(t):=\inf _{x, y \in B_{r},\|x-y\|=t, \alpha \in(0,1)}( & (\alpha f(x)+(1-\alpha) f(y) \\
& -f(\alpha x+(1-\alpha) y)) \\
& \left.\times(\alpha(1-\alpha))^{-1}\right),
\end{aligned}
$$

for all $t \geq 0$. The function $\rho_{r}$ is called the gauge of uniform convexity of $f$. The function $f$ is also said to be uniformly smooth on bounded subsets of $E$ if and only if $f^{*}$ is uniformly convex on bounded subsets of $E$.

In the sequel, we will need the following lemmas.

Lemma 7 (see [26]). Let $r>0$ be a constant and let $f: E \rightarrow$ $\mathbb{R}$ be uniformly convex on bounded subsets of $E$. Then,

$$
f\left(\sum_{k=0}^{n} \alpha_{k} x_{k}\right) \leq \sum_{k=0}^{n} \alpha_{k} f\left(x_{k}\right)-\alpha_{i} \alpha_{j} \rho_{r}\left(\left\|x_{i}-y_{j}\right\|\right),
$$

for all $i, j \in\{0,1,2, \ldots, n\}, x_{k} \in B r, \alpha_{k} \in(0,1)$, and $k=$ $0,1,2, \ldots, n$, with $\sum_{k=0}^{n} \alpha_{k}=1$, where $\rho_{r}$ is the gauge of uniform convexity of $f$.

Let $f: E \rightarrow(-\infty,+\infty)$ be a Gâteaux differentiable function. The modulus of total convexity of $f$ at $x \in \operatorname{dom}$ $f$ is the function $v_{f}(x,):.[0,+\infty) \rightarrow[0,+\infty]$ defined by

$$
v_{f}(x, t):=\inf \left\{D_{f}(y, x): y \in \operatorname{dom} f,\|y-x\|=t\right\} .
$$

The function $f$ is called totally convex at $x$ if $\nu_{f}(x, t)>0$, whenever $t>0$. The function $f$ is called totally convex if it is totally convex at any point $x \in \operatorname{int}(\operatorname{dom} f)$ and is said to be totally convex on bounded sets if $v_{f}(B, t)>0$ for any nonempty bounded subset $B$ of $E$ and $t>0$, where the modulus of total convexity of the function $f$ on the set $B$ is the function $\nu_{f}$ : $\operatorname{int}(\operatorname{dom} f) \times[0,+\infty) \rightarrow[0,+\infty]$ defined by

$$
v_{f}(B, t):=\inf \left\{V_{f}(x, t): x \in B \cap \operatorname{dom} f\right\} .
$$

We know that $f$ is totally convex on bounded sets if and only if $f$ is uniformly convex on bounded sets (see [32, Theorem 2.10]). The next lemma will be useful in the proof of our main results.

Lemma 8 (see [27]). Let $f: E \rightarrow(-\infty,+\infty]$ be a proper, lower semicontinuous, and convex function; then, for all $z \in E$, one has

$$
D_{f}\left(z, \nabla f^{*}\left(\sum_{i=1}^{N} t_{i} \nabla f\left(x_{i}\right)\right)\right) \leq \sum_{i=1}^{N} t_{i} D_{f}\left(z, x_{i}\right) .
$$

Lemma 9 (see [33]). Let $f: E \rightarrow \mathbb{R}$ be Gâteaux differentiable on $\operatorname{int}(\operatorname{dom} f)$ such that $\nabla f^{*}$ is bounded on bounded subsets of $\operatorname{dom} f^{*}$. Let $x^{*} \in X$ and $\left\{x_{n}\right\} \subset E$. If $\left\{D_{f}\left(x, x_{n}\right)\right\}$ is bounded, so is the sequence $\left\{x_{n}\right\}$.

Lemma 10 (see [32]). Let $C$ be a nonempty, closed, and convex subset of $E$. Let $f: E \rightarrow \mathbb{R}$ be a Gâteaux differentiable and totally convex function and let $x \in E$. Then,

(i) $z=P_{C}^{f}(x)$ if and only if $\langle\nabla f(x)-\nabla f(z), y-z\rangle \leq$ $0, \forall y \in C$;

(ii) $D_{f}\left(y, P_{C}^{f}(x)\right)+D_{f}\left(P_{C}^{f}(x), x\right) \leq D_{f}(y, x), \forall y \in C$.

Lemma 11 (see [34]). Let $f: E \rightarrow(-\infty,+\infty]$ be uniformly Fréchet differentiable and bounded on bounded sets of $E$; then $\nabla f$ is uniformly continuous on bounded subsets of $E$ from the strong topology of $E$ to the strong topology of $E^{*}$.

Let $f: E \rightarrow \mathbb{R}$ be a Legendre and Gâteaux differentiable function. Following $[2,3]$, we make use of the function $V_{f}$ : $E \times E^{*} \rightarrow[0,+\infty)$ associated with $f$, which is defined by

$V_{f}\left(x, x^{*}\right)=f(x)-\left\langle x, x^{*}\right\rangle+f^{*}\left(x^{*}\right), \quad \forall x \in E, x^{*} \in E^{*}$.

Then, $V_{f}$ is nonnegative and

$$
V_{f}\left(x, x^{*}\right)=D_{f}\left(x, \nabla f^{*}\left(x^{*}\right)\right) \quad \forall x \in E, x^{*} \in E^{*} .
$$

Moreover, by the subdifferential inequality,

$$
V_{f}\left(x, x^{*}\right)+\left\langle y^{*}, \nabla f^{*}\left(x^{*}\right)-x\right\rangle \leq V_{f}\left(x, x^{*}+y^{*}\right),
$$

$\forall x \in E$, and $x^{*}, y^{*} \in E^{*}$ (see [35]). 


\section{Main Result}

In the sequel, we will need the following lemma.

Lemma 12. Let $f: E \rightarrow \mathbb{R}$ be a Legendre function which is bounded, uniformly Fréchet differentiable, and totally convex on bounded subsets of $E$. For $p, x_{i} \in E(i=1,2, \ldots, N)$ and $\alpha_{i} \in[0,1]$ such that $\sum_{i=0}^{N} \alpha_{i}=1$ one has that

$$
\begin{gathered}
D_{f}\left(p, \nabla f^{*}\left(\alpha_{1} \nabla f\left(x_{1}\right)+\alpha_{2} \nabla f\left(x_{2}\right)+\cdots+\alpha_{N} \nabla f\left(x_{N}\right)\right)\right) \\
\leq \sum_{i=1}^{N} \alpha_{i} D_{f}\left(p, x_{i}\right)-\alpha_{i} \alpha_{j} \rho_{r}^{*}\left(\left\|\nabla f\left(x_{i}\right)-\nabla f\left(x_{j}\right)\right\|\right) .
\end{gathered}
$$

Proof. Since $f$ is uniformly Fréchet differentiable function we have by Lemma 11 that $\nabla f$ is uniformly continuous and hence by Theorem 3.6.3 of [31] we get that $f^{*}$ is uniformly convex on bounded subsets of $E$. This, with (24), (23), and Lemma 7, give that

$$
\begin{aligned}
D_{f} & \left(p, \nabla f^{*}\left(\alpha_{1} \nabla f\left(x_{1}\right)+\alpha_{2} \nabla f\left(x_{2}\right)+\cdots+\alpha_{N} \nabla f\left(x_{N}\right)\right)\right) \\
= & V_{f}\left(p, \alpha_{1} \nabla f\left(x_{1}\right)+\alpha_{2} \nabla f\left(x_{2}\right)+\cdots+\alpha_{N} \nabla f\left(x_{N}\right)\right) \\
= & f(p)-\left\langle p, \sum_{i=1}^{N} \alpha_{i} \nabla f\left(x_{i}\right)\right\rangle+f^{*}\left(\sum_{i=1}^{N} \alpha_{i} \nabla f\left(x_{i}\right)\right) \\
= & f(p)-\sum_{i=1}^{N} \alpha_{i}\left\langle p, \nabla f\left(x_{i}\right)\right\rangle+f^{*}\left(\sum_{i=1}^{N} \alpha_{i} \nabla f\left(x_{i}\right)\right) \\
\leq & f(p)-\sum_{i=1}^{N} \alpha_{i}\left\langle p, \nabla f\left(x_{i}\right)\right\rangle+\sum_{i=1}^{N} \alpha_{i} f^{*}\left(\nabla f\left(x_{i}\right)\right) \\
& -\alpha_{i} \alpha_{j} \rho_{r}^{*}\left(\left\|\nabla f\left(x_{i}\right)-\nabla f\left(x_{j}\right)\right\|\right) \\
= & \sum_{i=1}^{N} \alpha_{i} V_{f}\left(p, \nabla f\left(x_{i}\right)\right)-\alpha_{i} \alpha_{j} \rho_{r}^{*}\left(\left\|\nabla f\left(x_{i}\right)-\nabla f\left(x_{j}\right)\right\|\right) \\
= & \sum_{i=1}^{N} \alpha_{i} D_{f}\left(p, x_{i}\right)-\alpha_{i} \alpha_{j} \rho_{r}^{*}\left(\left\|\nabla f\left(x_{i}\right)-\nabla f\left(x_{j}\right)\right\|\right)
\end{aligned}
$$

for $i, j \in\{1,2, \ldots, N\}$. The proof is complete.

We now prove the following theorem.

Theorem 13. Let $f: E \rightarrow \mathbb{R}$ be a strongly coercive Legendre function which is bounded, uniformly Fréchet differentiable, and totally convex on bounded subsets of E. Let $C$ be a nonempty, closed, and convex subset of $\operatorname{int}(\operatorname{dom} f)$ and let $T_{i}$ : $C \rightarrow C$, for $i=1,2, \ldots, N$, be a finite family of Bregman weak relatively nonexpansive mappings. Assume that the interior of $F:=\bigcap_{i=1}^{N} F\left(T_{i}\right)$ is nonempty. For $x_{0} \in C$ let $\left\{x_{n}\right\}$ be a sequence generated by

$$
x_{n+1}=P_{C}^{f} \nabla f^{*}\left(\beta_{0} \nabla f\left(x_{n}\right)+\sum_{i=1}^{N} \beta_{i} \nabla f\left(T_{i} x_{n}\right)\right), \quad n \geq 0,
$$

where $\left\{\beta_{i}\right\}_{i=0}^{N} \subset[c, d] \subset(0,1)$ satisfy $\lim _{n \rightarrow \infty} \alpha_{n}=0$, $\sum_{n=1}^{\infty} \alpha_{n}=\infty$, and $\sum_{i=0}^{N} \beta_{i}=1$. Then, the sequence $\left\{x_{n}\right\}_{n \in \mathbb{N}}$ generated by (28) converges strongly to an element of $F$.

Proof. Let $p \in F$. Then, from (28), Lemma 10, and Lemma 12 we have that

$$
\begin{aligned}
D_{f}\left(p, x_{n+1}\right) & \\
= & D_{f}\left(p, P_{C}^{f} \nabla f^{*}\left(\beta_{0} \nabla f\left(x_{n}\right)+\sum_{i=1}^{N} \beta_{i} \nabla f\left(T_{i} x_{n}\right)\right)\right) \\
\leq & D_{f}\left(p, \nabla f^{*}\left(\beta_{0} \nabla f\left(x_{n}\right)+\sum_{i=1}^{N} \beta_{i} \nabla f\left(T_{i} x_{n}\right)\right)\right) \\
\leq & \beta_{0} D_{f}\left(p, x_{n}\right)+\sum_{i=1}^{N} \beta_{i} D_{f}\left(p, T_{i} x_{n}\right) \\
& -\beta_{0} \beta_{i} \sigma_{r}\left(\left\|T_{i} x_{n}-x_{n}\right\|\right) \\
\leq & \beta_{0} D_{f}\left(p, x_{n}\right)+\sum_{i=1}^{N} \beta_{i} D_{f}\left(p, x_{n}\right) \\
& -\beta_{0} \beta_{i} \sigma_{r}\left(\left\|T_{i} x_{n}-x_{n}\right\|\right) \\
\leq & D_{f}\left(p, x_{n}\right)-\beta_{0} \beta_{i} \sigma_{r}\left(\left\|T_{i} x_{n}-x_{n}\right\|\right) \leq D_{f}\left(p, x_{n}\right),
\end{aligned}
$$

for each $i \in\{1,2, \ldots, N\}$, and hence

$$
D_{f}\left(p, x_{n+1}\right) \leq D_{f}\left(p, x_{n}\right) .
$$

Therefore, $\lim _{n \rightarrow \infty} D_{f}\left(p, x_{n}\right)$ exists and hence by Lemma 9 we get that $\left\{x_{n}\right\}$ is bounded. Now, from (2), we also have that

$$
\begin{gathered}
D_{f}\left(p, x_{n}\right)-D_{f}\left(p, x_{n+1}\right)-D_{f}\left(x_{n+1}, x_{n}\right) \\
=\left\langle\nabla f\left(x_{n}\right)-\nabla f\left(x_{n+1}\right), x_{n+1}-p\right\rangle .
\end{gathered}
$$

This implies that

$$
\begin{aligned}
& \left\langle\nabla f\left(x_{n}\right)-\nabla f\left(x_{n+1}\right), x_{n+1}-p\right\rangle+D_{f}\left(x_{n+1}, x_{n}\right) \\
& =D_{f}\left(p, x_{n}\right)-D_{f}\left(p, x_{n+1}\right) .
\end{aligned}
$$

Since the interior of $F$ is nonempty, there exists $p^{*} \in F$ and $r>0$ such that $p^{*}+r h \in F$, whenever $\|h\| \leq 1$. Thus, from (30), we have that

$$
D_{f}\left(p^{*}+r h, x_{n+1}\right) \leq D_{f}\left(p^{*}+r h, x_{n}\right) .
$$

Therefore, from (32) and (33), we get that

$$
\begin{aligned}
0 \leq & \left\langle\nabla f\left(x_{n}\right)-\nabla f\left(x_{n+1}\right), x_{n+1}-\left(p^{*}+r h\right)\right\rangle \\
& +D_{f}\left(x_{n+1}, x_{n}\right) .
\end{aligned}
$$

Then, this and (32) imply that

$$
\begin{aligned}
r\langle\nabla f & \left.\left(x_{n}\right)-\nabla f\left(x_{n+1}\right), h\right\rangle \\
& \leq\left\langle\nabla f\left(x_{n}\right)-\nabla f\left(x_{n+1}\right), x_{n+1}-p^{*}\right\rangle+D_{f}\left(x_{n+1}, x_{n}\right) \\
& =\left(D_{f}\left(p^{*}, x_{n}\right)-D_{f}\left(p^{*}, x_{n+1}\right)\right)
\end{aligned}
$$


and hence

$$
\begin{aligned}
& \left\langle\nabla f\left(x_{n}\right)-\nabla f\left(x_{n+1}\right), h\right\rangle \\
& \quad \leq \frac{1}{r}\left(D_{f}\left(p^{*}, x_{n}\right)-D_{f}\left(p^{*}, x_{n+1}\right)\right) .
\end{aligned}
$$

Since $h$ with $\|h\| \leq 1$ is arbitrary, we have

$$
\left\|\nabla f\left(x_{n}\right)-\nabla f\left(x_{n+1}\right)\right\| \leq \frac{1}{r}\left(D_{f}\left(p^{*}, x_{n}\right)-D_{f}\left(p^{*}, x_{n+1}\right)\right) \text {. }
$$

So, if $n>m$, then

$$
\begin{aligned}
\left\|\nabla f\left(x_{m}\right)-\nabla f\left(x_{n}\right)\right\| \\
=\| \nabla f\left(x_{m}\right)-\nabla f\left(x_{m+1}\right)+\nabla f\left(x_{m+1}\right) \\
\quad-\cdots-\nabla f\left(x_{n-1}\right)+\nabla f\left(x_{n-1}\right)-\nabla f\left(x_{n}\right) \| \\
\leq \sum_{i=m}^{n-1}\left\|\nabla f\left(x_{i}\right)-\nabla f\left(x_{i+1}\right)\right\| \\
\leq \frac{1}{r} \sum_{i=m}^{n-1}\left(D_{f}\left(p^{*}, x_{i}\right)-D_{f}\left(p^{*}, x_{i+1}\right)\right) \\
=\frac{1}{r}\left(D_{f}\left(p^{*}, x_{m}\right)-D_{f}\left(p^{*}, x_{n}\right)\right) .
\end{aligned}
$$

We know that $\left\{\phi\left(\left(p^{*}, x_{n}\right)\right\}\right.$ converges. So, $\left\{\nabla f\left(x_{n}\right)\right\}$ is a Cauchy sequence. Since $E^{*}$ is complete there exists $y \in E^{*}$ such that $\left\{\nabla f\left(x_{n}\right)\right\}$ converges strongly to $y$. Furthermore, since $f$ is Legendre there exists $x^{*} \in E$ such that $y=\nabla f\left(x^{*}\right)$ and hence

$$
\nabla f\left(x_{n}\right) \longrightarrow \nabla f\left(x^{*}\right) \quad \text { as } n \longrightarrow \infty \text {. }
$$

Now, since $f$ is strongly coercive and uniformly convex on bounded subsets of $E, f^{*}$ is uniformly Fréchet differentiable and bounded on bounded subsets of $E$ (see [31, Prop. 3.6.2]). Thus, applying Lemma 11 we get that $x_{n}=\nabla f^{*}\left(\nabla f\left(x_{n}\right)\right) \rightarrow$ $x^{*}=\nabla f^{*}\left(\nabla f\left(x^{*}\right)\right)$, as $n \rightarrow \infty$.

Moreover, since $\left\{x_{n}\right\}$ is subset of $C$, which is closed and convex, we have that $x^{*} \in C$. that

Now, we show that $x^{*} \in \bigcap_{i=1}^{N} F\left(T_{i}\right)$. But from (29) we have

$$
\beta_{0} \beta_{i} \sigma_{r}\left(\left\|T_{i} x_{n}-x_{n}\right\|\right) \longrightarrow 0 \quad \text { as } n \longrightarrow \infty
$$

for each $i \in\{1,2, \ldots, N\}$. Then, the property of $\sigma_{r}$ implies that $\left\|T_{i} x_{n}-x_{n}\right\| \rightarrow 0$ as $n \rightarrow \infty$. From the fact that $T_{i}$, for each $i \in\{1,2, \ldots, N\}$, is Bregman weak relatively nonexpansive we obtain that $x^{*} \in F\left(T_{i}\right)$, for each $i \in\{1,2, \ldots, N\}$, and hence $x^{*} \in \bigcap_{i=1}^{N} F\left(T_{i}\right)$. This completes the proof.

If, in Theorem 13, every $T_{i}$ for each $i \in\{1,2, \ldots, N\}$ is Bregman relatively nonexpansive we have $\widehat{F}\left(T_{i}\right)=\widetilde{F}\left(T_{i}\right)=$ $F\left(T_{i}\right)$ for each $i=1,2, \ldots, N$. Thus, we get the following corollary.

Corollary 14. Let $f: E \rightarrow \mathbb{R}$ be a strongly coercive Legendre function which is bounded, uniformly Fréchet differentiable, and totally convex on bounded subsets of E. Let $C$ be a nonempty, closed, and convex subset of $\operatorname{int}(\operatorname{dom} f)$ and let $T_{i}: C \rightarrow C$, for $i=1,2, \ldots, N$, be a finite family of Bregman relatively nonexpansive mappings. Assume that the interior of $\mathscr{F}:=\bigcap_{i=1}^{N} F\left(T_{i}\right)$ is nonempty. For $x_{0} \in C$ let $\left\{x_{n}\right\}$ be a sequence generated by (28). Then, the sequence $\left\{x_{n}\right\}_{n \in \mathbb{N}}$ converges strongly to an element of $\mathscr{F}$.

If, in Theorem 13, we take $T_{i}$, for each $i=1,2, \ldots, N$, to be continuous Bregman quasi-nonexpansive mappings, then we have $\widetilde{F}\left(T_{i}\right)=F\left(T_{i}\right)$ for each $i=1,2, \ldots, N$. Thus, we have the following corollary.

Corollary 15. Let $f: E \rightarrow \mathbb{R}$ be a strongly coercive Legendre function which is bounded, uniformly Fréchet differentiable, and totally convex on bounded subsets of E. Let $C$ be a nonempty, closed, and convex subset of $\operatorname{int}(\operatorname{dom} f)$ and let $T_{i}: C \rightarrow C$, for $i=1,2, \ldots, N$, be a finite family of continuous Bregman quasi-nonexpansive mappings. Assume that the interior of $\mathscr{F}:=\bigcap_{i=1}^{N} F\left(T_{i}\right)$ is nonempty. For $x_{0} \in C$ let $\left\{x_{n}\right\}$ be a sequence generated by (28). Then, the sequence $\left\{x_{n}\right\}_{n \in \mathbb{N}}$ converges strongly to an element of $\mathscr{F}$.

If, in Theorem 13, we take $N=1$, then we have the following corollary.

Corollary 16. Let $f: E \rightarrow \mathbb{R}$ be a strongly coercive Legendre function which is bounded, uniformly Fréchet differentiable, and totally convex on bounded subsets of $E$. Let $C$ be a nonempty, closed, and convex subset of int $(\operatorname{dom} f)$ and let $T$ : $C \rightarrow C$ be a finite family of Bregman relatively nonexpansive mappings. Assume that the interior of $F(T)$ is nonempty. For $x_{0} \in C$ let $\left\{x_{n}\right\}$ be a sequence generated by

$$
x_{n+1}=P_{C}^{f} \nabla f^{*}\left(\beta \nabla f\left(x_{n}\right)+(1-\beta) \nabla f\left(T x_{n}\right)\right), \quad n \geq 0,
$$

where $\beta \in(0,1),\left\{\alpha_{n}\right\} \subset(0,1)$ satisfy $\lim _{n \rightarrow \infty} \alpha_{n}=0$ and $\sum_{n=1}^{\infty} \alpha_{n}=\infty$. Then, the sequence $\left\{x_{n}\right\}_{n \in \mathbb{N}}$ generated by (41) converges strongly to an element of $\mathscr{F}$.

Remark 17. Our results are new even if the convex function $f$ is chosen to be $f(x)=(1 / p)\|x\|^{p}(1<p<\infty)$ in uniformly smooth and uniformly convex spaces.

Remark 18. Our theorems improve and unify most of the results that have been proved for these important classes of nonlinear operators. In particular, Theorem 3.2 extends Theorem 3.1 of [23], Theorem 1 of [24], and Theorem 3.1 of [7] in the sense that either our theorem is applicable to a more general class of a finite family of Bregman weak relatively nonexpansive mappings or our scheme does not require the computation of $C_{n}$ or $Q_{n}$ for each $n \geq 0$ provided that the interior of $\mathscr{F}$ is nonempty.

Moreover, we observe that Theorem 3.2 extends Theorem 3.1 of [26] in the sense that our scheme does not require the computations of $C_{n}$ for each $n \geq 0$ when we consider finite family of Bregman weak relatively nonexpansive mappings and the interior of $\mathscr{F}$ is nonempty. 


\section{Disclosure}

The first author undertook this work when he was visiting the Abdus Salam International Centre for Theoretical Physics (ICTP), Trieste, Italy, as a regular associate.

\section{Conflict of Interests}

The authors declare that there is no conflict of interests regarding the publication of this paper.

\section{References}

[1] L. M. Brègman, "A relaxation method of finding a common point of convex sets and its application to the solution of problems in convex programming," USSR Computational Mathematics and Mathematical Physics, vol. 7, pp. 200-217, 1967.

[2] Y. Censor and A. Lent, "An iterative row-action method for interval convex programming," Journal of Optimization Theory and Applications, vol. 34, no. 3, pp. 321-353, 1981.

[3] Y. I. Alber, "Metric and generalized projection operators in Banach spaces: properties and applications," in Theory and Applications of Nonlinear Operators of Accretive and Monotone Type, vol. 178 of Lecture Notes in Pure and Applied Mathematics, pp. 15-50, Dekker, New York, NY, USA, 1996.

[4] S. Reich, "A weak convergence theorem for the alternating method with Bregman distances," in Theory and Applications of Nonlinear Operators of Accretive and Monotone Type, vol. 178 of Lecture Notes in Pure and Applied Mathematics, pp. 313-318, Marcel Dekker, New York, NY, USA, 1996.

[5] S. Reich and S. Sabach, "Two strong convergence theorems for a proximal method in reflexive Banach spaces," Numerical Functional Analysis and Optimization, vol. 31, no. 1-3, pp. 2244, 2010.

[6] S. Reich and S. Sabach, "Existence and approximation of fixed points of Bregman firmly nonexpansive mappings in reflexive Banach spaces," in Fixed-Point Algorithms for Inverse Problems in Science and Engineering, vol. 49, pp. 301-316, Springer, New York, NY, USA, 2011.

[7] J. Chen, Z. Wan, L. Yuan, and Y. Zheng, "Approximation of fixed points of weak Bregman relatively nonexpansive mappings in Banach spaces," International Journal of Mathematics and Mathematical Sciences, vol. 2011, Article ID 420192, 23 pages, 2011.

[8] C. Byrne, "A unified treatment of some iterative algorithms in signal processing and image reconstruction," Inverse Problems, vol. 20, no. 1, pp. 103-120, 2004.

[9] C. I. Podilchuk and R. J. Mammone, "Image recovery by convex projections using a least-squares constraint," The Journal of the Optical Society of America A, vol. 7, pp. 517-521, 1990.

[10] H. Zegeye and N. Shahzad, "Strong convergence theorems for monotone mappings and relatively weak nonexpansive mappings," Nonlinear Analysis. Theory, Methods \& Applications A, vol. 70, no. 7, pp. 2707-2716, 2009.

[11] H. Zegeye and N. Shahzad, "Viscosity approximation methods for a common fixed point of finite family of nonexpansive mappings," Applied Mathematics and Computation, vol. 191, no. 1, pp. 155-163, 2007.

[12] H. Zegeye and N. Shahzad, "A hybrid scheme for finite families of equilibrium, variational inequality and fixed point problems,"
Nonlinear Analysis. Theory, Methods \& Applications A, vol. 74, no. 1, pp. 263-272, 2011.

[13] H. Zegeye and N. Shahzad, "Strong convergence theorems for a solution of finite families of equilibrium and variational inequality problems," Optimization, vol. 63, no. 2, pp. 207-223, 2014.

[14] H. Zegeye and N. Shahzad, "Convergence theorems for a common point of solutions of equilibrium and fixed point of relatively nonexpansive multivalued mapping problems," Abstract and Applied Analysis, vol. 2012, Article ID 859598, 16 pages, 2012.

[15] W. R. Mann, "Mean value methods in iteration," Proceedings of the American Mathematical Society, vol. 4, pp. 506-510, 1953.

[16] S. Ishikawa, "Fixed points by a new iteration method," Proceedings of the American Mathematical Society, vol. 44, pp. 147-150, 1974.

[17] H. Zegeye and N. Shahzad, "Approximating common solution of variational inequality problems for two monotone mappings in Banach spaces," Optimization Letters, vol. 5, no. 4, pp. 691704, 2011.

[18] H. Zegeye and N. Shahzad, "A hybrid approximation method for equilibrium, variational inequality and fixed point problems," Nonlinear Analysis. Hybrid Systems, vol. 4, no. 4, pp. 619-630, 2010.

[19] H. Zegeye, "Strong convergence theorems for maximal monotone mappings in Banach spaces," Journal of Mathematical Analysis and Applications, vol. 343, no. 2, pp. 663-671, 2008.

[20] H. Zegeye and N. Shahzad, "Strong convergence theorems for a finite family of nonexpansive mappings and semigroups via the hybrid method," Nonlinear Analysis. Theory, Methods \& Applications A, vol. 72, no. 1, pp. 325-329, 2010.

[21] H. Zegeye, E. U. Ofoedu, and N. Shahzad, "Convergence theorems for equilibrium problem, variational inequality problem and countably infinite relatively quasi-nonexpansive mappings," Applied Mathematics and Computation, vol. 216, no. 12, pp. 3439-3449, 2010.

[22] K. Nakajo and W. Takahashi, "Strong convergence theorems for nonexpansive mappings and nonexpansive semigroups," Journal of Mathematical Analysis and Applications, vol. 279, no. 2, pp. 372-379, 2003.

[23] S. Matsushita and W. Takahashi, "A strong convergence theorem for relatively nonexpansive mappings in a Banach space," Journal of Approximation Theory, vol. 134, no. 2, pp. 257-266, 2005.

[24] S. Reich and S. Sabach, "A projection method for solving nonlinear problems in reflexive Banach spaces," Journal of Fixed Point Theory and Applications, vol. 9, no. 1, pp. 101-116, 2011.

[25] S. Reich and S. Sabach, "Two strong convergence theorems for Bregman strongly nonexpansive operators in reflexive Banach spaces," Nonlinear Analysis. Theory, Methods \& Applications A, vol. 73, no. 1, pp. 122-135, 2010.

[26] E. Naraghirad and J.-C. Yao, "Bregman weak relatively nonexpansive mappings in Banach spaces," Fixed Point Theory and Applications, vol. 2013, article 141, 2013.

[27] R. R. Phelps, Convex Functions, Monotone Operators and Differentiability, vol. 1364 of Lecture Notes in Mathematics, Springer, Berlin, Germany, 2nd edition, 1993.

[28] H. H. Bauschke, J. M. Borwein, and P. L. Combettes, "Essential smoothness, essential strict convexity, and Legendre functions in Banach spaces," Communications in Contemporary Mathematics, vol. 3, no. 4, pp. 615-647, 2001. 
[29] J. F. Bonnans and A. Shapiro, Perturbation Analysis of Optimization Problems, Springer, New York, NY, USA, 2000.

[30] J.-B. Hiriart-Urruty and C. Lemaréchal, Convex Analysis and Minimization Algorithms II, vol. 306 of Grundlehren der Mathematischen Wissenschaften, Springer, Berlin, Germany, 1993.

[31] C. Zălinescu, Convex Analysis in General Vector Spaces, World Scientific, River Edge, NJ, USA, 2002.

[32] D. Butnariu and E. Resmerita, "Bregman distances, totally convex functions, and a method for solving operator equations in Banach spaces," Abstract and Applied Analysis, vol. 2006, Article ID 84919, 39 pages, 2006.

[33] V. Martín-Márquez, S. Reich, and S. Sabach, "Bregman strongly nonexpansive operators in reflexive Banach spaces," Journal of Mathematical Analysis and Applications, vol. 400, no. 2, pp. 597614, 2013.

[34] S. Reich and S. Sabach, "A strong convergence theorem for a proximal-type algorithm in reflexive Banach spaces," Journal of Nonlinear and Convex Analysis, vol. 10, no. 3, pp. 471-485, 2009.

[35] F. Kohsaka and W. Takahashi, "Proximal point algorithms with Bregman functions in Banach spaces," Journal of Nonlinear and Convex Analysis, vol. 6, no. 3, pp. 505-523, 2005. 


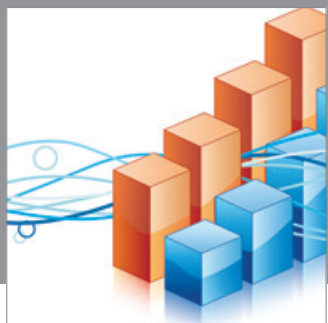

Advances in

Operations Research

mansans

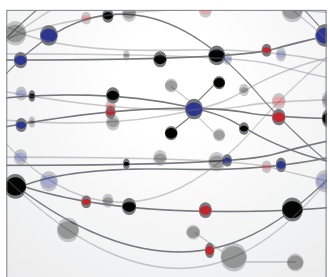

The Scientific World Journal
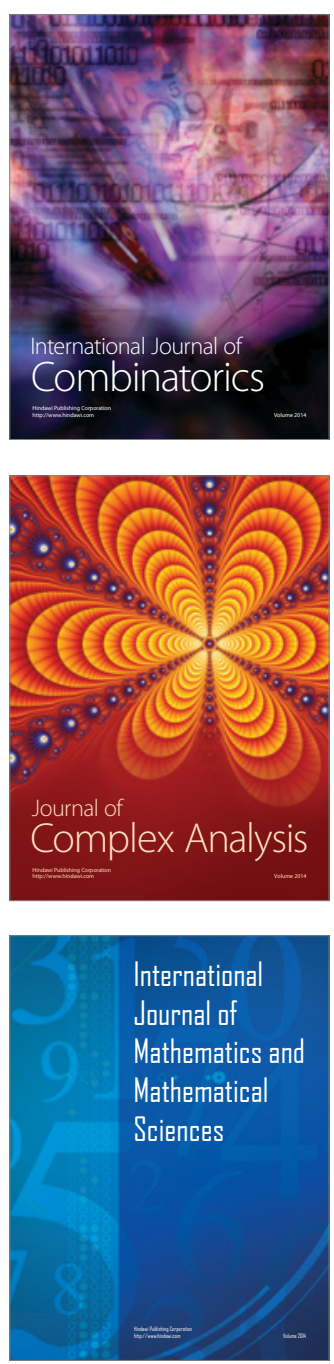
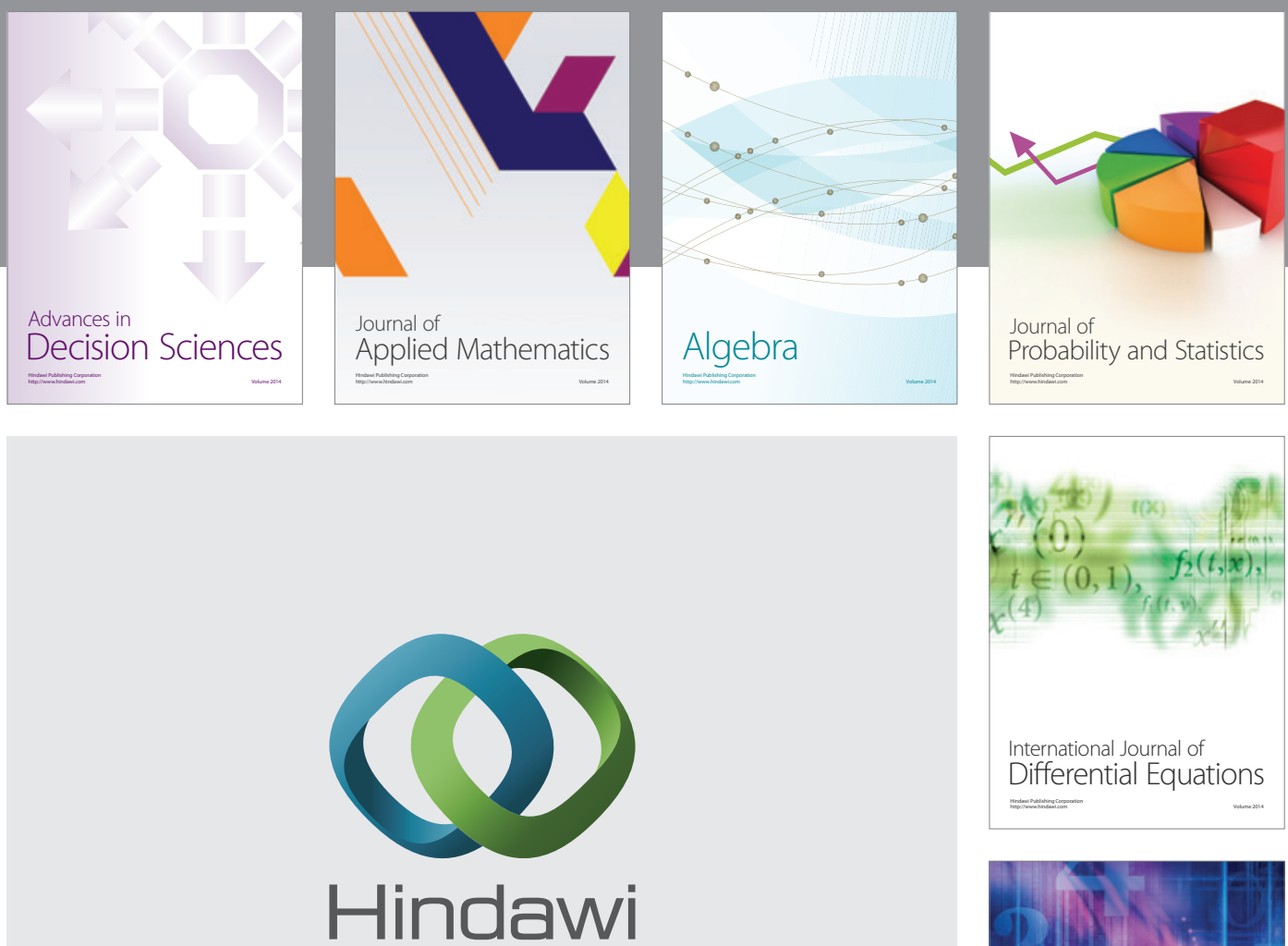

Submit your manuscripts at http://www.hindawi.com
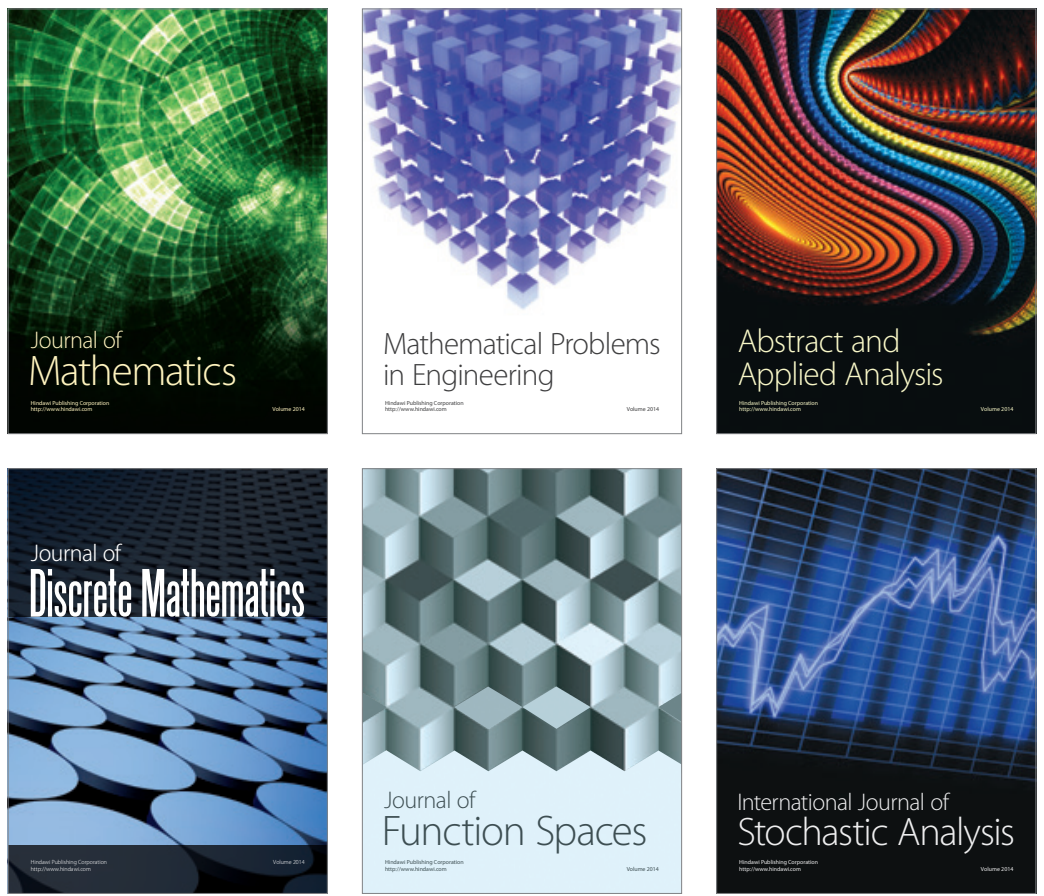

Journal of

Function Spaces

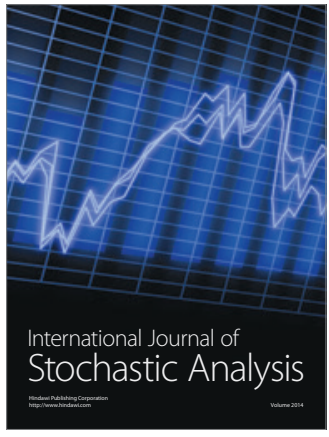

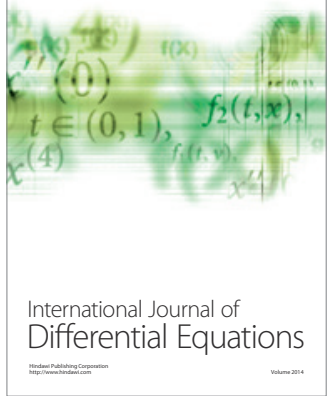
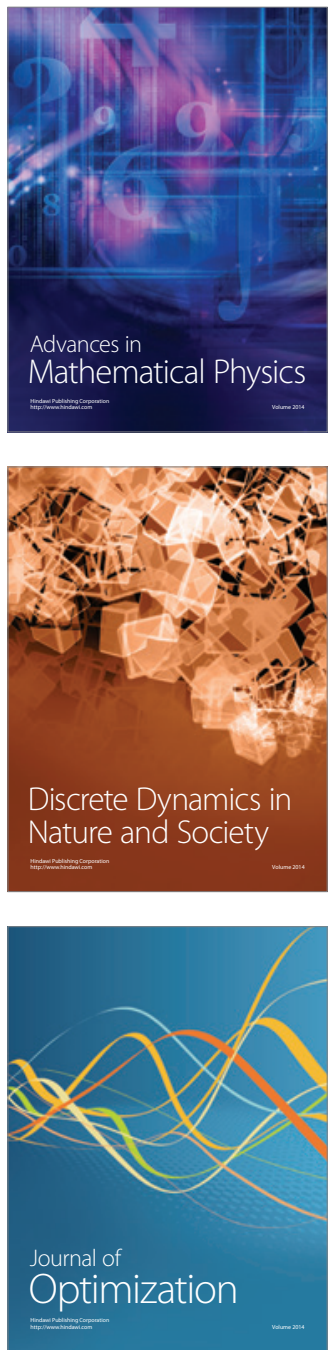